\title{
Impurity Scattering from $\delta$-layers in Giant Magnetoresistance Systems
}

\author{
C.H. Marrows* and B.J. Hickey \\ Department of Physics and Astronomy, E.C. Stoner Laboratory, University of Leeds, Leeds. LS2 9JT United Kingdom
}

(November 11, 2018)

\begin{abstract}
The properties of the archetypal $\mathrm{Co} / \mathrm{Cu}$ giant magnetoresistance (GMR) spin-valve structure have been modified by the insertion of very thin (sub-monolayer) $\delta$-layers of various elements at different points within the Co layers, and at the $\mathrm{Co} / \mathrm{Cu}$ interface. Different effects are observed depending on the nature of the impurity, its position within the periodic table, and its location within the spin-valve. The GMR can be strongly enhanced or suppressed for various specific combinations of these parameters, giving insight into the microscopic mechanisms giving rise to the GMR.
\end{abstract}

75.70.Pa, 75.70.-i, 73.20.At

Ever since the development of the first transistor, solidstate science and technology has sought a proper description of the details of electronic transport in heterostructures. The past few years has seen a remarkably high level of activity in the area of magnetic heterostructures on the nanometer scale, not least in the area of giant magnetoresistance (GMR) [1], observed in ultrathin layered structures featuring transition metal ferromagnets that can have the relative orientation of their layer moments altered by a magnetic field. The broad physical picture describing GMR is that it arises from spin-dependent scattering, so that parallel or antiparallel magnetic layer moments correspond to aligned or antialigned filters for spin-polarized current. Approaches to the theory based on the Boltzmann formalism [2, 3] can give a good phenomenological description of the basic effects. Early quantum pictures used a free-electron-like band, evaluating the Kubo formula for the case of spindependent scattering potentials [A. [5]. More recent theoretical treatments have emphasized the importance of the electronic structure to the GMR [6 10], which yield a better quantitative agreement with experiment.

Nevertheless these theories only consider pairs of materials $(e . g . \mathrm{Fe} / \mathrm{Cr}, \mathrm{Co} / \mathrm{Cu})$, limiting the understanding of more complex experimental structures. One area of contention is the microscopic location of the spin-dependent scattering - in the bulk or at the interface of the ferromagnetic layers. It has been attempted to get directly at the microscopic origin of the GMR by deliberately doping with impurities. This was reported for $\mathrm{Fe} / \mathrm{Cr}$ multilayers using a few different dopants placed at the interface [11, 12]. The different impurities have different values for the scattering spin-asymmetry $\alpha$, defined as the ratio of spin $\uparrow$ to spin $\downarrow$ scattering from the impurity $\rho_{\downarrow} / \rho_{\uparrow} 13$ 15, an essentially phenomenological parameter - only in the last few years have attempts been made to determine $\alpha$ from from electronic band structure calculations [16]. Similar interfacial doping experiments were reported by Shinjo [17]. Nevertheless these experiments were carried out using AF coupled superlattices, complicating the interpretation, as the AF state is ill-defined, and is easily degraded by the insertion of the dopants, leading to a loss of GMR merely due to loss of AF alignment [18]. Meanwhile Vouille et al. have studied the effects of doping various elements into the magnetic layers as alloys [19] - although this varied the $\alpha$ of the dopants, determining the relative bulk and interface contributions of these scatterers is model dependent.

A noteworthy theoretical treatment of the both the position and spin asymmetry $(\alpha)$ properties of impurities in $\mathrm{Co} / \mathrm{Cu}$ multilayers has been given by Zahn et al. [20]. Using the tight-binding Korringa-Kohn-Rostoker technique they were able to calculate the local density of states [21] and hence the effect of the impurity scatterers on the GMR. In this way direct conclusions can be drawn about the relative importance of bulk and interface scattering - however these ideas have not been tested at all stringently by any of the experiments cited above.

In this Letter we wish to address these issues, reporting on experiments in which we have systematically doped archetypal $\mathrm{Co} / \mathrm{Cu}$ spin-valves by the insertion of $\delta$-layers of various elements to localize scattering with a certain value of $\alpha$. The use of spin-valves removes the difficulties in ensuring a proper AF alignment, as we always have a clear distinction between parallel $(\uparrow \uparrow)$ and antiparallel $(\uparrow \downarrow)$ moment alignments - so we can be certain to have measured the full GMR amplitude, defined as $\left(\rho_{\uparrow \downarrow}-\rho_{\uparrow \uparrow}\right) / \rho_{\uparrow \uparrow}$. The previous experiments used only a few impurities, we have prepared a much larger set of samples to systematically study the dependence of the GMR on the changes in electronic structure caused by the introduction of a wide variety of different dopants. In addition our $\delta$-doping technique yields important information on the position dependence of the impurities that cannot be obtained by forming alloys or interfacial layers alone. We have observed long ranged interactions between several different impurities and the interfacial spin-dependent scattering, over distances up to an order of magnitude greater than those previously reported 22] or predicted [23].

The samples were deposited by dc magnetron sputtering in a computer controlled custom vacuum system with a base pressure of $2 \times 10^{-8}$ Torr. The substrates were pieces cut from (001) Si wafer, the working gas was 

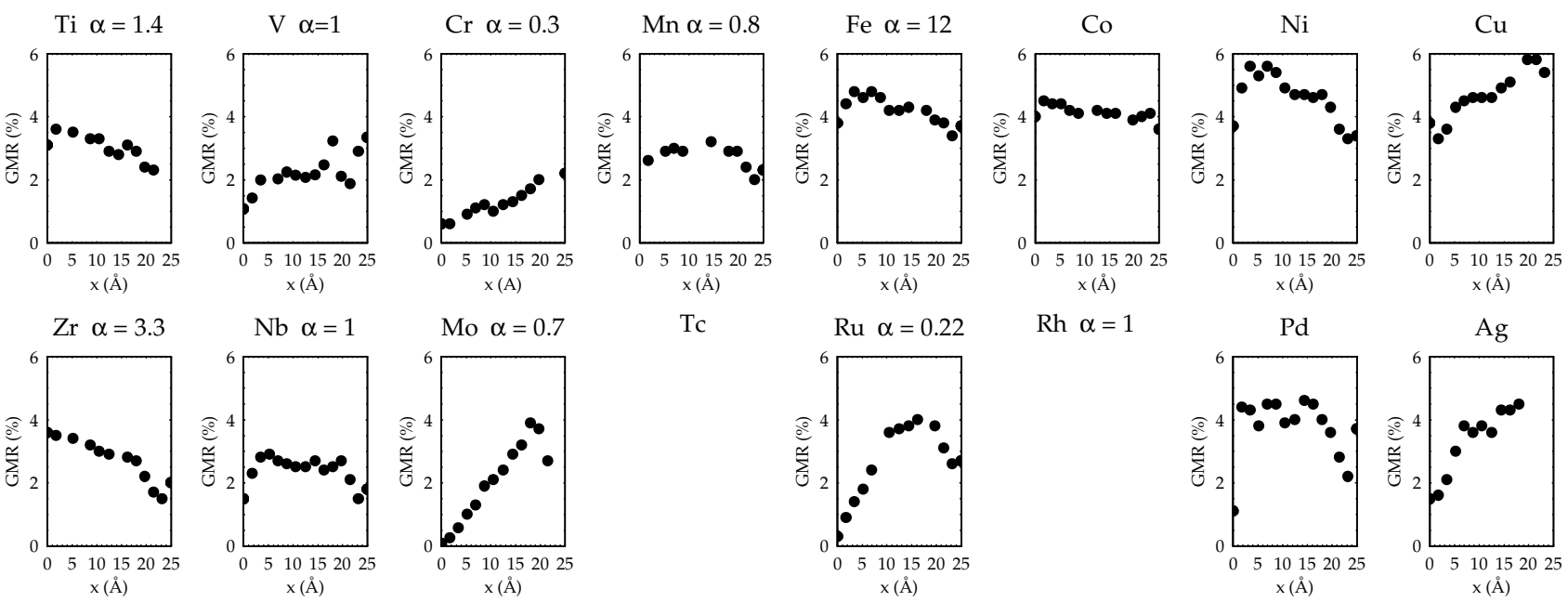

Tc

$\mathrm{Ru} \alpha=0.22$

$\mathrm{Rh} \alpha=1$
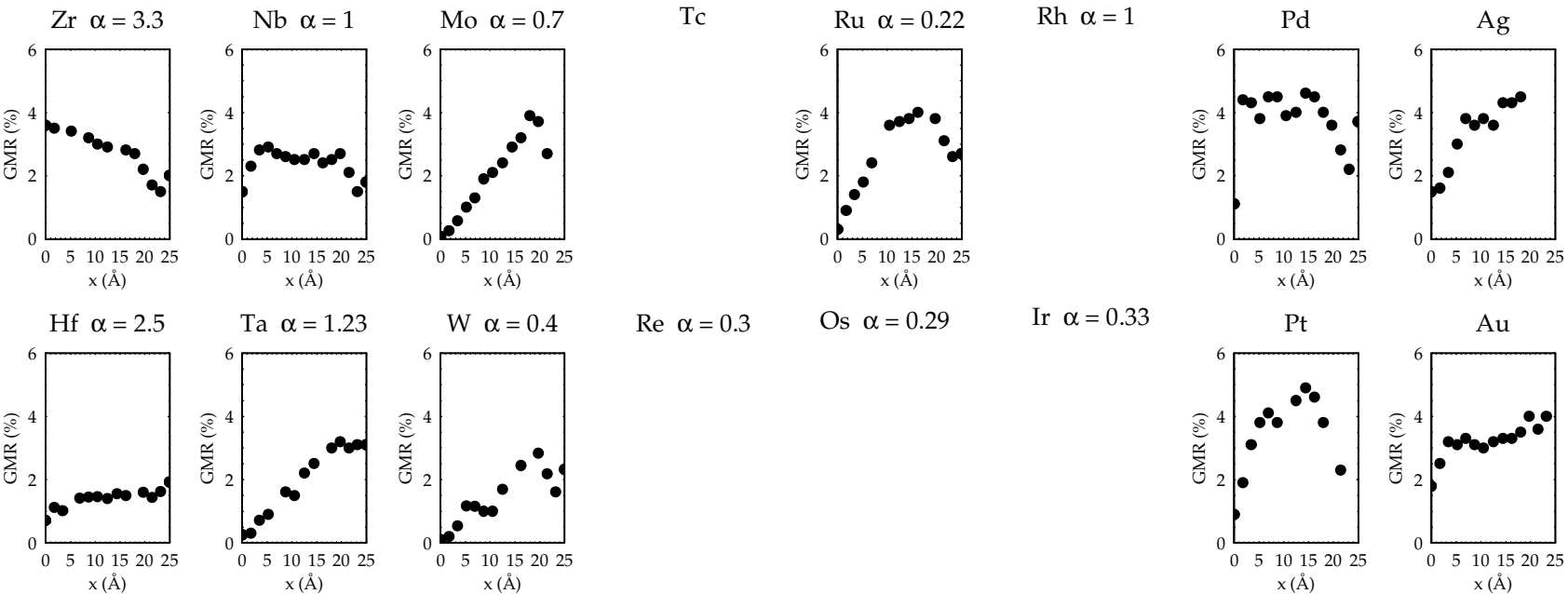

Os $\alpha=0.29$

Ir $\alpha=0.33$
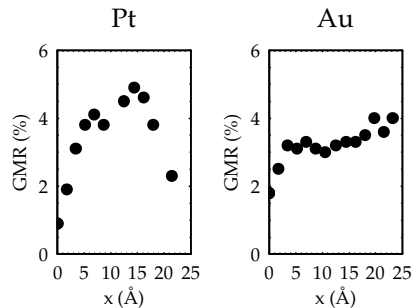

FIG. 1. Position dependence of the giant magnetoresistance for various transition metal impurities in the Co layer of the spin-valves. Elements for which $\alpha$ values are available from reference 13 have these values noted at the top of each panel. The graph width represents the Co layer thickness. As $x$ increases in each graph we move from the $\mathrm{Co} / \mathrm{Cu}$ interface to outermost surface of the Co layers. 
3.0 mTorr of Ar, and typical deposition rates were $\sim 3$ $\AA s^{-1}$. The substrates are heat-sunk during deposition so that the temperature does not rise by more than a few ${ }^{\circ} \mathrm{C}$ above ambient. Magnetoresistance was measured by a standard dc 4-probe method, at room temperature. The sample structure comprises those elements found in a typical spin-valve - two Co layers separated by $\mathrm{Cu}$, with an FeMn pinning layer. The impurity $\delta$-layer is inserted into the Co at different points so that the overall structure is as follows: Si substrate / $\mathrm{Ta}(50) / \mathrm{Co}(25-x) / \mathrm{X}$ / $\mathrm{Co}(x) / \mathrm{Cu}(30) / \mathrm{Co}(x) / \mathrm{X} / \mathrm{Co}(25-x) / \operatorname{FeMn}(80)$ / Ta(25); all thicknesses are given in A. Since both the Ta and FeMn have resistivities of much greater than 100 $\mu \Omega \mathrm{cm}$, we should expect most of the in-plane conduction to take place in the GMR active $\mathrm{Co} / \mathrm{Cu} / \mathrm{Co}$ sandwich. In all cases the amount of impurity corresponds to a few tenths of a monolayer - we used standard conditions of $0.5 \mathrm{~s}$ deposition using a power density of 1 $\mathrm{W} / \mathrm{cm}^{2}$. In some cases the introduction of the $\delta$-layer close to the $\mathrm{Co} / \mathrm{FeMn}$ interface reduced the exchange bias to the point where the $\uparrow \downarrow$ state cannot be accessed, such data points have been omitted from all the Figures that we present.

Structural changes have been noted in similar experiments: the use of sub-monolayer amounts of impurities as surfactants 24,25] can change the resistivity as they alter growth modes whilst floating out of the film on the growth front. We have tested for such effects and not found them: there is little change in the observed GMR if we restrict ourselves to a $\delta$-layer in only one or other Co layer. Since the $\delta$ layer is being moved, in sequential samples, in opposite directions through the stack in these two cases the effects cannot be due to changes due to its floating out, as surfactant effects can only occur in layers deposited after the $\delta$-layer.

In Fig. 11 the GMR is plotted against the position of the dopant $\delta$-layer for a variety of elements from the central part of the transition metal block. Firstly the reader should note that the graph for Co is quite flat at $\sim 4.5 \%$, and this can be regarded as the control experiment - a $\delta$-layer of Co was inserted into both Co layers. This modest value is due to the thinness of the Co layers compared to those used in device applications [26]. It is immediately evident that it is not possible to increase the GMR of a $\mathrm{Co} / \mathrm{Cu}$ structure by putting any other impurity at the interface, previously thought to be that part of the structure most susceptible to changes in chemical species 22.

On the other hand, certain impurities will increase the GMR when placed within the Co, contrary to commonly held views about the pre-eminence of the interfaces for GMR. The neighboring plots for Ni and Fe show a similar behavior - both curves show a pronounced rise in GMR when the $\delta$-layer is placed just behind, but not at, the interface. The effect is larger for $\mathrm{Ni}-$ almost $50 \%$ higher. One possible interpretation is that the $\delta$-layer forms a second highly effective spin-filter just behind the first filter of the $\mathrm{Co} / \mathrm{Cu}$ interface. Although no value is reported in Ref. [13] for $\mathrm{Ni}$, the value of $\alpha_{\mathrm{Fe}}=12$ given for Fe in Co leads one to suppose that the value of $\alpha_{\mathrm{Ni}}$ must also be $\gg 1$, and likely to be even higher still than the value for Fe.

The effects of $\mathrm{Cu}$ impurities are also of particular interest. When close to the interface there is little effect, or a small suppression, due presumably to the artificial creation of a more interdiffused, alloyed interfacial layer. However, once the $\mathrm{Cu}$ is deep inside the layer we see an enhanced GMR, somewhat unexpected in the light of the fact that these are non-magnetic impurities. An obvious comparison here is with the large bulk spin-anisotropy in the resistivity of $\mathrm{Ni}$ layers doped with $\mathrm{Cu}$ observed by Vouille et al. 19.

Within the group of noble metals, the GMR is lower for $\mathrm{Ag}$ impurities than for $\mathrm{Cu}$, and lower still for $\mathrm{Au}$. The behavior is consistent with greater spin-orbit scattering - the heavier elements flip spins more readily, mixing the spin current channels. A comparison with, for example, $\mathrm{Pd}$ and $\mathrm{Pt}$ is consistent; the GMR recovers more rapidly as $\mathrm{Pd}$ is moved away from the $\mathrm{Co} / \mathrm{Cu}$ interface. Both these elements, with strong Stoner susceptibility enhancements in the bulk, are readily polarized by the Co matrix, leading to little loss in GMR.

On the other hand the graphs for $\mathrm{Cr}, \mathrm{Mo}, \mathrm{Ru}$, Ta and $\mathrm{W}$ all show that the insertion of the $\delta$-layer at the interface almost totally suppresses the GMR. As the impurity is moved back into the Co the GMR rises in a roughly linear fashion. For Ru and Ta the GMR appears to plateau when the dopant is $\sim 10$ and $20 \AA$ from the interface respectively. This is exactly the behavior expected given the importance attached to interfacial scattering, but the length scale is greater than that of only $\sim 2.5 \AA$ previously reported when $\mathrm{Co} \delta$-layers were inserted into $\mathrm{NiFe}$ [22], suggesting that the lengthscales involved in discussions of interfacial or bulk scattering must be highly material system dependent. For all of these materials but Ta, the reported $\alpha$ value is $<1$. The value of $\alpha_{\mathrm{Ta}}=1.23$ appears to be an overestimate.

The data for $\mathrm{Mn}, \mathrm{V}$ and $\mathrm{Nb}$ also look similar. These elements have $\alpha$ values reported $\simeq 1$, and we see that the dependence on the position of the dopant layer is quite weak. The GMR is suppressed wherever the $\delta$ layer is placed. There is little or no suppression of the GMR when the elements $\mathrm{Ti}$ or $\mathrm{Zr}$, both with $\alpha>1$, are introduced into the interfacial region of the Co layer. The effects of Hf are anomalous in this regard, possibly either $\alpha_{\mathrm{Hf}}=2.5$ is an over-estimate, or the high nuclear charge of Hf leads to a large spin-orbit scattering term. This is to be compared with the results found for Ta.

It is also of interest to pose the question regarding the effects of impurities in the $\mathrm{Cu}$ spacer layer. The reader's attention is drawn to Fig. 2, where the GMR of spinvalves with $\mathrm{Co}(\mathrm{Cu})$ impurities in the $\mathrm{Cu}(\mathrm{Co})$ layer(s) is 


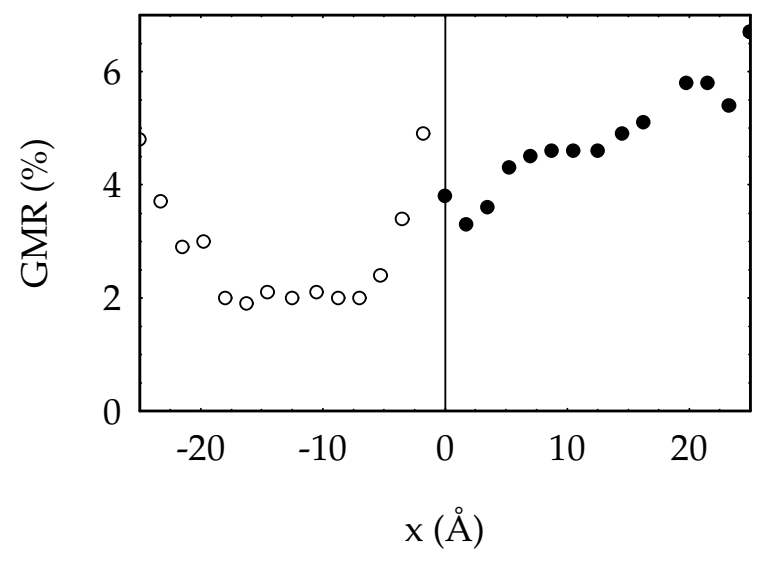

FIG. 2. Dependence of the GMR on the position of Co impurities in $\mathrm{Cu}$ (open symbols) or $\mathrm{Cu}$ impurities in $\mathrm{Co}$ (solid symbols). $x=0$ corresponds to the position of the $\mathrm{Cu} / \mathrm{Co}$ interface.

presented. The data for the $\mathrm{Cu}$ impurities (solid symbols) is taken from Fig. 1. As we have seen, the GMR rises as the $\mathrm{Cu}$ moves back into the Co after a small suppression close to the interface. On the other hand Co impurities in the $\mathrm{Cu}$ spacer strongly reduce the GMR with only a weak position dependence unless they are close to the interface. We should expect that Co atoms or clusters isolated in the $\mathrm{Cu}$ should behave (super)paramagnetically, leading to spin-independent scattering when averaging over time or position in the film, as in practical measurements. The decay length of $\sim 10 \AA$ is therefore a direct measure of the range of significant exchange interactions for the Co impurities in $\mathrm{Cu}$. Further experiments with other impurities in the spacer layer are all consistent with the same general picture - a position independent suppression of the GMR due to a shortening of the mean free path in the crucial spacer layer, unless the impurity is within two or three atomic sites of the interfacial region, where the impurity can begin to affect nature of the interfacial scattering.

We find that the experimental results are at odds with the published theoretical predictions of Zahn et al. 20] in the following important ways: impurities with $\alpha<1$ suppress the GMR, usually to a great extent when at the interface, and still have a considerable effect when several lattice constants away from the interface; impurities with $\alpha>1$ sometimes do provide an enhancement of the GMR, but it is only to be found when they are a few $\AA$ behind the $\mathrm{Co} / \mathrm{Cu}$ interface; and impurities in the spacer layer have a dramatic effect by lowering the GMR. There are two omissions in the theory of Zahn et al. which may lead to inaccurate predictions: a lack of interband transitions, found to have an important effect on conductivity calculations when realistic levels of disorder are included [9]; and vertex corrections are required for an accurate description of impurity scattering [27].
The results of more sophisticated calculations by Binder et al. [28], are qualitatively much more in accord with the observations that we report here. Selfconsistently calculated impurity potentials were used, as well as a more correct description of the microscopic transport processes including state-dependent relaxation times and proper account taken of the scattering-in term. In particular the predictions of the change in GMR when moving the $\delta$-layers of specific materials from the interface in to the bulk of the Co show remarkable similarities with the observations and the sign of this change exhibits strong correlations with the sign of the exchange interaction calculated between the local moment of the impurity ion and the Co matrix.

The comprehensive nature of the data set allows some general conclusions to be drawn - there are consistent trends in the data for impurity $\delta$-dopants with $\alpha<1$, $\simeq 1$, and $>1$. The position dependence of the scattering that leads to the GMR has been shown to be remarkably rich, and has important implications for what is meant when bulk or interface scattering is discussed. There are three rather striking results, deserving of theoretical explanation: the opposite slopes of the data for $\alpha>1$ or $<1$, as exemplified by $\mathrm{Zr}$ and Mo; the significant increase in GMR caused by the insertion of $3 d$ ferromagnet dopants behind the $\mathrm{Co} / \mathrm{Cu}$ interface; and the marked increase in GMR when a nonmagnetic impurity, $\mathrm{Cu}$, is embedded deep in the bulk of the Co. As well as suggesting possible routes to optimizing GMR materials for devices, any theory found to be capable of reproducing all these effects must contain the correct physics of GMR at a deep level.

We should like to express our gratitude to J. Binder, P. Zahn and I. Mertig for thought-provoking discussions and access to unpublished theoretical results. We should also like to thank E.Yu. Tsymbal for helpful comments and suggestions. C.H. Marrows would like to thank the Royal Commission for the Exhibition of 1851 for financial support.

* Email: c.marrows@leeds.ac.uk

[1] A. Barthélémy and A. Fert in Handbook of Magnetic Materials, edited by K.H.J. Buschow, (North-Holland, Amsterdam, 1999).

[2] R.E. Camley and J. Barnas, Phys. Rev. Lett. 63(6), 664 (1989).

[3] B. Dieny, J. Phys.: Cond. Matt. 4, 8009 (1992).

[4] P.M. Levy, S. Zhang, and A. Fert, Phys. Rev. Lett 65, 1643, (1990); S. Zhang, P.M. Levy, and A. Fert, Phys. Rev. B 45, 8689, (1992).

[5] A. Vedyayev, B. Dieny, and N. Ryshnova, Europhys. Lett. 19, 329, (1992). 
[6] K.M. Schep, P.J. Kelly, and G.E.W. Bauer, Phys. Rev. Lett. 74, 586 (1995).

[7] W.H. Butler, X.-G. Zhang, D.M.C. Nicholson, T.C. Schulthess, and J.M. Maclaren, Phys. Rev. Lett. 76, 3216 (1996).

[8] W.H. Butler, X.-G. Zhang, D.M.C. Nicholson, and J.M. MacLaren. Phys. Rev. B 52, 13399 (1995).

[9] E.Yu. Tsymbal and D.G. Pettifor, Phys. Rev. B 54(21), 15314 (1996).

[10] S. Sanvito, C.J. Lambert, J.H. Jefferson, and A.M. Bratkovsky, Phys. Rev. B 59, 11936, (1999).

[11] P. Baumgart, B.A. Gurney, D.R. Wilhoit, T. Nguyen, B. Dieny, and V.S. Speriosu, J. Appl. Phys. 69, 4792, (1991).

[12] B.A. Gurney, P. Baumgart, D.R. Wilhoit, B. Dieny, and V.S. Speriosu, J. Appl. Phys. 70, 5867, (1991).

[13] H.A.M. van den Berg in Magnetic Multilayers and Giant Magnetoresistance, edited by U. Hartmann, (Springer, Berlin, 1999), p. 215.

[14] I.A. Campbell and A. Fert, in Ferromagnetic Materials, edited by E.P. Wohlfarth (North-Holland, Amsterdam, 1982), Vol. 3, p. 747.

[15] We would wish to note that the values for the spinasymmetry parameter given in the previous two references show considerable experimental scatter and moreover are for the specific case of dilute impurities in a bulk host. However they can at least be taken as a general guide.

[16] V.Yu. Irkhin and Yu.P. Irkhin, J. Magn. Magn. Mater 164, 119 (1996).

[17] T. Shinjo, Thin Solid Films 281-282, 469, (1996).

[18] We have found this to be the case in our laboratory; M. Perez, C.H. Marrows, and B.J. Hickey, (unpublished).

[19] C. Vouille, A. Barthélémy, F.E. Mpondo, A. Fert, P.A. Schroeder, S.Y. Hsu, A. Reilly, and R. Loloee, Phys. Rev. B 60, 6710 (1999).

[20] P. Zahn, J. Binder, I. Mertig, R. Zeller and P.H. Dederichs, Phys. Rev. Lett. 80, 4309, (1998); in this paper our parameter $\alpha$ is denoted by the symbol $\beta$.

[21] J. Binder, P. Zahn, I. Mertig, R. Zeller and P.H. Dederichs, Philos. Mag. B 78, 537, (1998).

[22] S.S.P. Parkin, Phys. Rev. Lett. 71, 1641 (1993).

[23] M.A.M. Gijs and G.E.W. Bauer, Adv. Phys 46, 285 (1997).

[24] R. Miranda , Physica Scripta T49B, 579 (1993).

[25] W.F. Egelhoff, P.J. Chen PJ, C.J. Powell, M.D. Stiles, and R.D. McMichael, J. Appl. Phys. 79, 2491 (1996); W.F. Egelhoff, P.J. Chen, C.J. Powell, M.D. Stiles, R.D. McMichael, C.L. Lin, J.M. Sivertsen, J.H. Judy, K. Takano, and A.E Berkowitz, J. Appl. Phys. 80, 5183 (1996).

[26] We have prepared spin-valves with up to 10\% GMR for devices: F.E. Stanley, C.H. Marrows, and B.J. Hickey, J. Appl. Phys. 87, 4864 (2000).

[27] J.C. Swihart, W.H. Butler, G.M. Stocks, D.M. Nicholson, and R.C. Ward, Phys. Rev. Lett. 57, 1181, (1986).

[28] Preliminary results are reported in J. Binder, P. Zahn and I. Mertig, J. Appl. Phys. 87, 5182 (2000). 